\title{
Dexmedetomidine-Tiletamine-Zolazepam Followed by Inhalant Anesthesia in Spectacled Bears (Tremarctos ornatus)
}

\author{
Natache Arouca Garofalo ${ }^{1}$, André Augusto Justo², Stephanie Cristine Miyamoto Araújo ${ }^{3}$, \\ Mayara Travalini de Lima ${ }^{4}$, Carlos Roberto Teixeira ${ }^{3}$ \& Francisco José Teixeira Neto ${ }^{3}$
}

\begin{abstract}
Background: The spectacled bear (Tremarctos ornatus) is the only bear species inhabiting South America and is classified as vulnerable according to the International Union for Conservation of Nature (IUCN) Red List of Threatened Species. Among the few publications on the use of general anesthesia and advanced monitoring of ursids in veterinary hospital settings, little is described regarding chemical restraint, general anesthesia and monitoring of spectacled bears. This case series describes the use of a dexmedetomidine-tiletamine-zolazepam chemical restraint combination and its effects on cardiorespiratory variables and arterial blood gases observed in two spectacled bears undergoing isoflurane anesthesia for imaging and/or surgical procedures.

Cases: Two female, one adult and one senile, all-term captive spectacled bears were referred to the Veterinary Teaching Hospital at the Universidade Estadual Paulista - Unesp, Botucatu campus, both with a presumable history of recent trauma. After immobilization with an intramuscular (IM) administration of tiletamine-zolazepam $(3.8-4.3 \mathrm{mg} / \mathrm{kg})$ and dexmedetomidine $(6.4-7.6 \mu \mathrm{g} / \mathrm{kg})$, induction of anesthesia was achieved by means of intravenous (IV) propofol $(1-2 \mathrm{mg} / \mathrm{kg})$. After orotracheal intubation animals underwent isoflurane anesthesia under mechanical ventilation through the remainder of the procedures. Initial settings of inspiratory flow rate were adjusted to obtain peak airway pressure $\left(\mathrm{P}_{\text {peak }}\right)$ of $10 \mathrm{cmH}_{2} \mathrm{O}$ and tidal volumes $(\mathrm{Vt})$ of $10 \mathrm{~mL} / \mathrm{kg}$, as well as respiratory rates $(f \mathrm{R})$ and inspiration-to-expiration (I:E) ratio of $10 \mathrm{breaths} / \mathrm{min}$ and 1:2, respectively, and were then adjusted throughout anesthesia to maintain normocapnia (end-tidal carbon dioxide concentrations between 35 and $45 \mathrm{mmHg})$. One of the individuals was chemically restrained $(6.4 \mathrm{mg} / \mathrm{kg}$ of tiletaminezolazepam and $7.7 \mu \mathrm{g} / \mathrm{kg}$ of dexmedetomidine) on a second anesthetic event for imaging procedures. Arterial blood gas analysis were performed with animals breathing room air and oxygen-enriched air. Both animals exhibited severe hypoxemia (partial pressure of oxygen $\left[\mathrm{PaO}_{2}\right]<60 \mathrm{mmHg}$ ) while breathing room air (inspired oxygen fraction $\left[\mathrm{FiO}_{2}\right] \cong 0.21$ ). An impaired blood oxygenation $\left(\mathrm{PaO}_{2} / \mathrm{FiO}_{2}<400\right)$ was still observed despite mechanical ventilation and the provision of $1.0 \mathrm{FiO}_{2}$. Alveolar recruitment maneuvers (3 sequential mechanical sights with $\mathrm{P}_{\text {peak }}$ at $20-30 \mathrm{cmH}_{2} \mathrm{O}$ during $15-30 \mathrm{~s}$ each) were then performed, which resulted in improved $\mathrm{PaO}_{2} / \mathrm{FiO}_{2}$ ratios. All other blood gas, electrolytes and acid-base variables did not appear to be importantly altered by chemical restraint and general anesthesia.

Discussion: In spite of severe hypoxemia recorded in animals breathing room air, dexmedetomidine-tiletamine-zolazepam resulted in reliable chemical restraints and is a feasible option for immobilizing spectacled bears. Hypoxemia is the most commonly described complication in bear anesthesia, and was also evidenced in the present report. However, low $\mathrm{PaO}_{2} / \mathrm{FiO}_{2}$ ratios tend to be paralleled by hypoventilation and therefore counteracted by oxygen supplementation in bears, which was not observed in the present report. In fact, blood oxygenation only reached acceptable values after alveolar recruitment maneuvers, which is compatible with an atelectasis-related hypoxemia. Ideally, inhalant anesthesia or field chemical restraint should be accompanied by advanced monitoring (cardiorespiratory variables and blood gas analysis) until further studies address the management of hypoxemia in spectacled bear. Advanced monitoring was of major importance for a safe outcome and an uneventful recovery in this species.
\end{abstract}

Keywords: balanced anesthesia, general anesthesia, spectacled bear, Tremarctos ornatus, wildlife.

${ }^{1}$ Veterinary Medical Teaching Hospital \& ${ }^{3}$ Department of Veterinary Surgery and Animal Reproduction, School of Veterinary Medicine and Animal Science, São Paulo State University (UNESP), Botucatu, SP, Brazil. ${ }^{2}$ Department of Surgery, School of Veterinary Medicine and Animal Science, University of São Paulo (USP), São Paulo, SP. ${ }^{4}$ Department of Anesthesiology, Medical School - UNESP, Botucatu. CORRESPONDENCE: N.A. Garofalo [natache.garofalo@unesp.br]. Veterinary Medical Teaching Hospital, School of Veterinary Medicine and Animal Science - UNESP. Rua Prof. Dr. Walter Mauricio Correa s/n. CEP 18618-681 Botucatu, São Paulo, Brazil. 


\section{INTRODUCTION}

The spectacled bear (Tremarctos ornatus), also known as Andean bear, is the only extant ursid species endemic to South America and is typically found along the Andes Mountains [26]. In spite of its wide habitat range, the species is classified as "vulnerable" regarding its global extinction risk, since habitat encroachment, climate change and the expansion of road systems have altogether fragmented the spectacled bear's territory and reduced its abundance [26].

All bear species must undergo chemical restraint followed or not by general anesthesia for wildlife management or for ordinary care in zoo practices [5]. Likewise the majority of wild species, the scientific literature on bear anesthesia has mainly focused on describing out-of-hospital chemical restraint protocols for non-invasive procedures [2-7,10-13,22,25], with few studies addressing general anesthesia and advanced monitoring in ursids $[15,20,24]$. Although chemical immobilization has been described for polar [2], brown [3,5,10-13,25], black [4,7], and sun bears [22], anesthesia in Tremarctos ornatus is still in its infancy [20]. Inasmuch as the threats to native populations of spectacled bears have grown in recent years, there is an increased need to develop safe anesthetic combinations and monitoring in this species. The purpose of this report is to describe the chemical restraint and the cardiorespiratory changes recorded during inhalant anesthesia in two spectacled bears.

\section{CASES}

Case 1. An 8-year-old adult female spectacled bear (Tremarctos ornatus), inhabitant of the Zoological Park "Quinzinho de Barros" (Sorocaba, São Paulo, Brazil), was found limping and was referred to the Veterinary Medical and Research Centre in Wild Animals (CEMPAS) at the São Paulo State University (UNESP), School of Veterinary Medicine and Animal Science (Botucatu, SP, Brazil).

Upon admission, the animal showed lameness of the pelvic limb. Body weight was estimated at $90 \mathrm{~kg}$ and chemical immobilization was performed with intramuscular (IM) tiletamine-zolazepam ${ }^{1}$ combined with dexmedetomidine ${ }^{2}$ at target doses of $3 \mathrm{mg} / \mathrm{kg}$ and $5 \mu \mathrm{g} / \mathrm{kg}$, respectively. A pressurized gas system using carbon dioxide deployed a dart at close range by means of a blowpipe. Complete immobilization was achieved within 10 minutes. After actual body weight was $70 \mathrm{~kg}$, doses of tiletamine-zolazepam ${ }^{1}$ and dexmedetomidine ${ }^{2}$ effectively administered were $3.8 \mathrm{mg} / \mathrm{kg}$ and $6.4 \mu \mathrm{g} /$ $\mathrm{kg}$, respectively.

An 18 G intravenous (IV) catheter ${ }^{3}$ was aseptically inserted into the right saphenous vein before the animal was transferred to the surgical facility. Thirty seven min after blow darting, anesthesia was induced with IV propofol $^{4}(2 \mathrm{mg} / \mathrm{kg}$ ) and orotracheal intubation was performed with a $14.0 \mathrm{~mm}$ endotracheal tube ${ }^{5}$ upon direct visualization of the larynx with a laryngoscope blade. The bear was positioned in lateral recumbency and anesthesia was maintained with isoflurane ${ }^{6}$ diluted in 1.0 inspired oxygen fraction $\left(\mathrm{FiO}_{2}\right)$ at a flow rate of $50 \mathrm{~mL} / \mathrm{kg} / \mathrm{min}$ delivered through a circle breathing circuit. The vaporizer setting was initially adjusted to maintain immobility and prevent spontaneous breathing efforts during intermittent positive-pressure ventilation ${ }^{7}$. A lactated Ringer's solution $^{8}$ was administered at $5 \mathrm{~mL} / \mathrm{kg} / \mathrm{h}$ using a peristaltic pump ${ }^{9}$.

After the onset of mechanical ventilation, an arterial sample was obtained by puncture of the femoral artery to evaluate temperature-corrected blood gases and electrolytes ${ }^{10}$ [partial pressures of oxygen $\left(\mathrm{PaO}_{2}\right)$ and carbon dioxide $\left(\mathrm{PaCO}_{2}\right), \mathrm{pH}$, bicarbonate $\left(\mathrm{HCO}_{3}{ }^{-}\right)$, extracellular base excess $\left(\mathrm{BE}_{\text {ecf }}\right)$, anion gap (GAP), lactate, sodium $\left(\mathrm{Na}^{+}\right)$, potassium $\left(\mathrm{K}^{+}\right)$, chloride $\left(\mathrm{Cl}^{-}\right)$and hematocrit $\left.(\mathrm{Ht})\right]$. Twenty min after propofol administration, mild acidosis and a significantly low $\mathrm{PaO}_{2} / \mathrm{FiO}_{2}$ ratio (below 200) were observed (Table 1). An alveolar recruitment maneuver was then performed (3 sequential mechanical sights with peak airway pressure $\left[\mathrm{P}_{\text {peak }}\right]$ at $20-30 \mathrm{cmH}_{2} \mathrm{O}$ during $15-30 \mathrm{~s}$ each).

Cardiorespiratory variables were recorded by a multiparameter monitor ${ }^{11}$ at 5 min intervals throughout anesthesia. Although under continuous cardiorespiratory monitoring, the first set of physiological data was recorded only $50 \mathrm{~min}$ after darting, because of the time spent transferring the patient to the operating room. A pulse oximeter probe placed on the tongue recorded hemoglobin peripheral oxygen saturation $\left(\mathrm{SpO}_{2}\right)$. A multi-gas analyzer was connected to the Y-piece of the breathing circuit for continuous measurement of end-tidal carbon dioxide $\left(\mathrm{ETCO}_{2}\right)$ and end-tidal isoflurane concentrations $\left(\mathrm{ET}_{\mathrm{ISO}}\right)$ through a sidestream sampling line. Initial settings of inspiratory flow rate were adjusted to obtain $\mathrm{P}_{\text {peak }}$ of $10 \mathrm{cmH}_{2} \mathrm{O}$ and tidal volumes $(\mathrm{Vt})$ of $10 \mathrm{~mL} / \mathrm{kg}$. Respiratory rate $\left(f_{\mathrm{R}}\right)$ and 
inspiration-to-expiration (I:E) ratio were initially set at 10 breaths/min and 1:2, respectively, and were then adjusted throughout anesthesia to maintain $\mathrm{ETCO}_{2}$ between 35 - $45 \mathrm{mmHg}$.

Heart rate (HR) and rhythm were also recorded (lead II electrocardiogram). Core temperature was monitored by an esophageal sensor and maintained above $36.5^{\circ} \mathrm{C}$ by means of a forced warm air device ${ }^{12}$. Additionally, the right dorsal pedal artery was aseptically catheterized with a $20 \mathrm{G}$ catheter and connected via a noncompliant tubing to a pressure transducer system filled with heparinized (5 IU/mL) physiological saline ${ }^{13}$ to measure systolic, diastolic and mean arterial blood pressures (SAP, DAP and MAP, respectively). The arterial catheter was also used to collect samples for blood gas and electrolyte analysis throughout anesthesia (Figure 1). Although the first recruitment maneuver improved the $\mathrm{PaO}_{2} / \mathrm{FiO}_{2}$ ratio from 92 to 260 (Table 1) at 46 min of inhalant anesthesia, it remained below normal limits (400), therefore this maneuver was repeated. The third arterial blood sample was collected 120 min after the onset of anesthesia and revealed a normal $\mathrm{PaO}_{2} / \mathrm{FiO}_{2}$ ratio, therefore an oxygen-air mixture $\left(\mathrm{FiO}_{2} \cong 0.6\right)$ was thereafter delivered throughout the remainder of the procedure (Table 1).

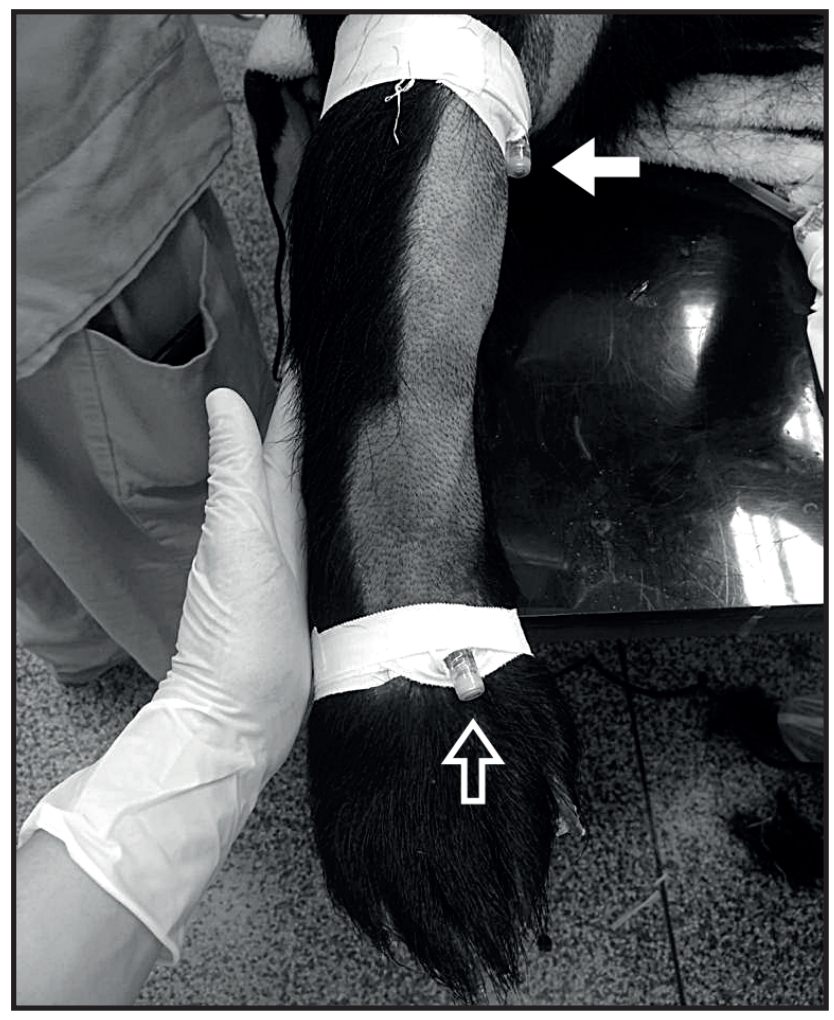

Figure 1. Catheterization of the dorsal pedal artery (open arrow) and the saphenous vein (bold arrow) in Case 1 bear.
Radiography revealed a tibial fracture and an osteosynthesis was performed with an intramedullary pin and a plate-rod fixation. With the aim of providing intraoperative analgesia, a combined sciatic and femoral nerve blocks ( $\mathrm{ScFeNB}$ ) were performed under ultrasound guidance in B-Mode with a 7.5 MHz linear array transducer ${ }^{14}$. Both techniques were accomplished by insertion of a $160 \times 2 \mathrm{~mm}$ Klein cannula and were adapted from recommendations in dogs [18]. Briefly, the sciatic nerve was approached in the middle third of thigh with the transducer positioned perpendicular to the long axis of the pelvic limb, caudal to the femur. The sciatic nerve was identified between the fascia of the biceps femoris and abductor muscles. At this site, a combination of $2 \%$ lidocaine $^{15}(2.8 \mathrm{mg} / \mathrm{kg})$ and $0.5 \%$ bupivacaine $^{6}(0.7 \mathrm{mg} / \mathrm{kg})$ was administered (total volume: $0.3 \mathrm{~mL} / \mathrm{kg}$ ). The femoral nerve blockade required a medial approach to the thigh (just above the femoral triangle), where a transverse image of the femoral artery, vein and nerve were obtained. This site received $0.1 \mathrm{~mL} / \mathrm{kg}$ of the $1: 1 \mathrm{mixture}$ of lidocaine $(1 \mathrm{mg} / \mathrm{kg})$ and bupivacaine $(0.25 \mathrm{mg} / \mathrm{kg})$. For additional analgesia, IV morphine ${ }^{6}(0.1 \mathrm{mg} / \mathrm{kg})$ and IV meloxicam ${ }^{16}$ $(0.1 \mathrm{mg} / \mathrm{kg})$ were also administered.

Adjustments in ventilator resulted in $\mathrm{ETCO}_{2}$ between 33 and $48 \mathrm{mmHg}$. No arrhythmias were noticed and $\mathrm{SpO}_{2}$ remained above $95 \%$. Direct blood pressure and HR were stable during the first hour of surgery, but gradually increased from that point onwar-

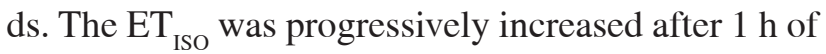
surgery by adjusting vaporizer settings to control the sympathetic response to surgical stimulation (Table 2 ). At the end of surgery, the patient was weaned from the ventilator by stepwise reducing $\mathrm{P}_{\text {peak }}$ and $f_{\mathrm{R}}$ until spontaneous respiratory efforts were evident on the capnographic waveform. Extubation was performed upon recovery of the swallowing and palpebral reflexes. Recovery from anesthesia was uneventful. Immediate postoperative pain assessment showed no signs of discomfort and no rescue analgesics were administered at this time point.

The bear was anesthetized a month later for surgical follow-up with tiletamine-zolazepam and dexmedetomidine $(6.4 \mathrm{mg} / \mathrm{kg}$ and $7.7 \mu \mathrm{g} / \mathrm{kg}$, respectively). Total dosages of drugs were higher than previously used in this animal because of poor dart placement. An arterial blood gas sample collected while the animal was breathing room air again showed mild acidosis and 
Table 1. Temperature $\left({ }^{\circ} \mathrm{C}\right)$ corrected partial pressures of oxygen $\left(\mathrm{PaO}_{2}\right)$ and carbon dioxide $\left(\mathrm{PaCO}_{2}\right)$, pH, bicarbonate $\left(\mathrm{HCO}_{3}^{-}\right)$, base excess of extracellular fluid $\left(\mathrm{BE}_{\text {ecf }}\right)$, anion gap $(\mathrm{AG})$, lactate, sodium $\left(\mathrm{Na}^{+}\right)$, potassium $\left(\mathrm{K}^{+}\right)$, chloride $\left(\mathrm{Cl}^{-}\right)$and hematocrit $(\mathrm{Ht})$ obtained either by puncture of the femoral artery or by the arterial line placed within the dorsal pedal artery of two spectacled bears submitted to different inspired oxygen fractions $\left(\mathrm{FiO}_{2}\right)$.

\begin{tabular}{|c|c|c|c|c|c|c|c|c|}
\hline \multirow{3}{*}{$\frac{\text { Variable }}{\text { Temperature }\left({ }^{\circ} \mathrm{C}\right)}$} & \multicolumn{5}{|c|}{ Bear 1} & \multirow{3}{*}{$\frac{\text { Bear 1* }}{\text { Puncture }}$} & \multicolumn{2}{|c|}{ Bear 2} \\
\hline & \multirow{2}{*}{$\frac{\text { Puncture }}{37.8}$} & \multicolumn{4}{|c|}{ Catheter } & & Puncture & Catheter \\
\hline & & 37.3 & 36.8 & 36.5 & 36.9 & & - & 37.1 \\
\hline$\cong \mathrm{FiO}_{2}(\%)$ & 1.0 & 1.0 & 1.0 & 0.6 & 0.6 & 0.21 & 0.21 & 1.0 \\
\hline $\mathrm{PaO}_{2}(\mathrm{mmHg})$ & 92 & 260 & 494 & 363 & 309 & 42 & 56 & 287 \\
\hline $\mathrm{PaO}_{2} / \mathrm{FiO}_{2}$ & $92^{\mathrm{a}}$ & $260^{\mathrm{b}}$ & $494^{c}$ & $605^{c}$ & $515^{\mathrm{c}}$ & 200 & $266^{\mathrm{d}}$ & $287^{\mathrm{a}}$ \\
\hline $\mathrm{PaCO}_{2}(\mathrm{mmHg})$ & 40.7 & 40 & 46.1 & 44.8 & 46 & 45.6 & 39.2 & 35.2 \\
\hline $\mathrm{pH}$ & 7.32 & 7.32 & 7.30 & 7.30 & 7.31 & 7.28 & 7.35 & 7.36 \\
\hline $\mathrm{HCO}_{3}^{-}(\mathrm{mmol} / \mathrm{L})$ & 20.1 & 20 & 22.1 & 21.6 & 22.3 & 20.6 & 21.1 & 19.5 \\
\hline $\mathrm{BE}_{\mathrm{ecf}}(\mathrm{mmol} / \mathrm{L})$ & -4.8 & -5.0 & -3.4 & -3.9 & -3.1 & -5.0 & -3.5 & -4.9 \\
\hline $\mathrm{AG}(\mathrm{mmol} / \mathrm{L})$ & 17.4 & 18 & 16.7 & 16.7 & 16.6 & 20.4 & 12.4 & 15.7 \\
\hline Lactate $(\mathrm{mmol} / \mathrm{L})$ & 0.4 & 0.2 & 0.1 & 0.3 & 0.2 & 0.7 & 0.4 & 0.4 \\
\hline $\mathrm{Na}^{+}(\mathrm{mmol} / \mathrm{L})$ & 149 & 151 & 146 & 148 & 149 & 143 & 145 & 150 \\
\hline $\mathrm{K}^{+}(\mathrm{mmol} / \mathrm{L})$ & 3.20 & 3.12 & 3.60 & 3.47 & 3.77 & 3.58 & 3.37 & 2.88 \\
\hline $\mathrm{Cl}^{-}(\mathrm{mmol} / \mathrm{L})$ & 111 & 113 & 107 & 110 & 110 & 102 & 111 & 115 \\
\hline $\mathrm{Ht}(\%)$ & 43 & 38 & 36 & 36 & 36 & 42 & 41 & 35 \\
\hline
\end{tabular}

*Second anesthetic procedure of Case 1 bear. ${ }^{a}$ After the onset of oxygen supplementation and mechanical ventilation. ${ }^{\mathrm{b}} \mathrm{After}$ the first recruitment maneuver. ${ }^{\mathrm{c}}$ After the second recruitment maneuver and onset of air-oxygen mixture. ${ }^{\mathrm{d} B e f o r e}$ oxygen supplementation and mechanical ventilation.

severe hypoxemia (Table 1). Because of the short time required for immobilization and rapid onset of recovery the animal did not receive supplemental oxygen.

Case 2. A 27-year-old senile female spectacled bear, from the "São Carlos Ecological Park" (São Carlos, São Paulo, Brazil) was admitted to the Veterinary Hospital because of a functional impotence of the right thoracic limb. Body weight was estimated at $60 \mathrm{~kg}$ and the animal was blowpipe-darted with tiletamine-zolazepam and dexmedetomidine mixture at target doses of $3 \mathrm{mg} / \mathrm{kg}$ and $5 \mu \mathrm{g} / \mathrm{kg}$, respectively. The bear briskly moved just prior to injection, which led to dart misplacement. Fifteen min later, the bear was not recumbent and approaching the animal was judged unsafe. Supplemental doses of tiletamine-zolazepam and dexmedetomidine ( $1 \mathrm{mg} / \mathrm{kg}$ and $2 \mu \mathrm{g} / \mathrm{kg}$, respectively) were administered at the thigh by blowpipe. Complete immobilization was achieved within $3 \mathrm{~min}$ of the second injection (18 min from initial darting). Actual body weight was $55 \mathrm{~kg}$, and doses effectively administered of tiletamine-zolazepam and dexmedetomidine were $4.3 \mathrm{mg} / \mathrm{kg}$ and $7.6 \mu \mathrm{g} / \mathrm{kg}$, respectively. While the animal was breathing room air, an arterial blood sample was collected by puncture of the femoral artery for blood gas and electrolyte analysis. All variables showed unremarkable changes, except for a severe hypoxemia $\left(\mathrm{PaO}_{2}<60 \mathrm{mmHg}\right)$ [Table 1]. In order to provide cardiorespiratory support and because a long period of immobilization was anticipated for diagnostic procedures, the animal underwent general anesthesia. Firstly, an $18 \mathrm{G}$ IV catheter was inserted into the right saphenous vein. General anesthesia was induced with IV propofol $(1 \mathrm{mg} / \mathrm{kg}$ ) and orotracheal intubation was performed exactly as described in Case 1 (Figure 2). Anesthesia was maintained with isoflurane in oxygen $\left(\mathrm{FiO}_{2}: 1.0\right.$; oxygen flow rate: $50 \mathrm{~mL} / \mathrm{kg} / \mathrm{min}$ ) delivered through a circle breathing circuit. Mechanical ventilation settings and monitored physiological variables followed the same pattern of Case 1. A lactated Ringer's solution ${ }^{8}$ was administered at $10 \mathrm{~mL} / \mathrm{kg} / \mathrm{h}$ using a peristaltic pump because the patient was mildly 


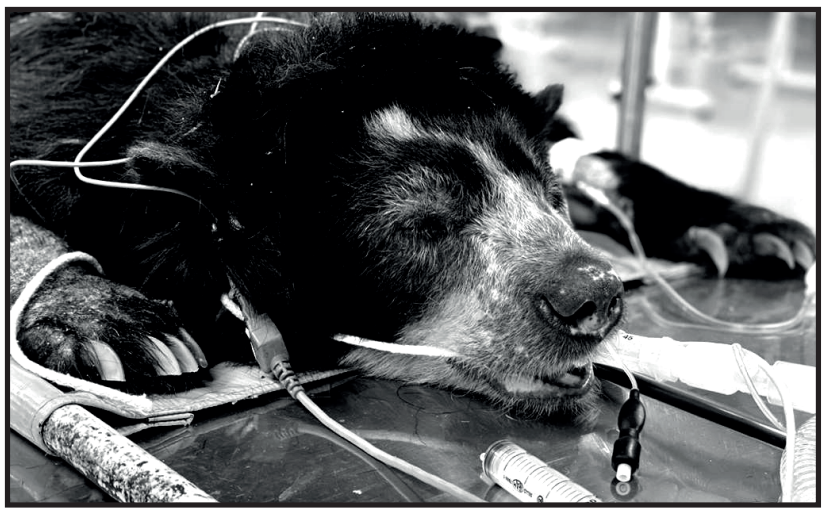

Figure 2. Case 2 bear submitted to general anesthesia.

dehydrated (estimated 5\% dehydration). Intravenous methadone ${ }^{6}(0.1 \mathrm{mg} / \mathrm{kg})$ and meloxicam ${ }^{16}(0.1 \mathrm{mg} / \mathrm{kg})$ were also provided for analgesia.

The $\mathrm{ET}_{\text {ISO }}$ was maintained at $1.1-1.7 \%$ throughout the procedure. The ventilator settings resulted in $\mathrm{ETCO}_{2}$ values ranging from 30 - $35 \mathrm{mmHg}$. Pulse oximetry, HR and arterial pressure were stable throughout anesthesia (Table 2). A second arterial blood sample collected from the arterial catheter $60 \mathrm{~min}$ after induction of anesthesia showed a relatively low $\mathrm{PaO}_{2} / \mathrm{FiO}_{2}$ ratio (Table 1) and the same recruitment maneuver described for Case 1 was performed.

At the end of the imaging procedures, isoflurane was discontinued ( $90 \mathrm{~min}$ after induction of anesthesia). The animal was weaned from mechanical ventilation and extubated as described in Case 1. The bear was conducted to its enclosure and recovery was uneventful.

\section{DISCUSSION}

Immobilization with tiletamine-zolazepam and dexmedetomidine was smooth and recumbency was achieved within $10 \mathrm{~min}$ of the last darting in both cases. Although vomiting, hypersalivation and seizure-like episodes are common complications in bear anesthesia $[5,6,10,22,25]$, these were not observed in the present report. The combination of tiletaminezolazepam and dexmedetomidine at the doses of this report provided good muscle relaxation and allowed safe handling the bears. The onset of immobilization from the last darting $(<10 \mathrm{~min})$ was similar to that reported for spectacled bears anesthetized with tiletamine-zolazepam-medetomidine (8 min) [20]. Brief onsets of immobilization augment the safety of wildlife procedures because it enables an early approach and anesthetic monitoring [1].
Dexmedetomidine probably improved muscle relaxation and reduced tiletamine-zolazepam requirements for immobilization, since doses were at least twice as low in comparison to doses of tiletamine-zolazepam alone described in captive [2] and in free-ranging bears [3]. A recent review on spectacled bear anesthesia revealed that tiletamine-zolazepam $(1.5 \mathrm{mg} / \mathrm{kg})$, ketamine $(1.9 \mathrm{mg} / \mathrm{kg})$ and medetomidine $(15 \mu \mathrm{g} / \mathrm{kg})$ were the most commonly reported combinations for chemical restraint, yet $48 \%$ of animals required supplemental injections for proper immobilization [20]. Despite the higher doses of tiletamine-zolazepam reported here (mean $4.1 \mathrm{mg} / \mathrm{kg}$ ), the combination with dexmedetomidine resulted in a reliable chemical restraint without adverse effects. Dexmedetomidine doses were within the range of those reported for other bear species $[7,10,25]$. The chemical restraint protocol at dosages reported here could be used for minor procedures in zoo practices or as premedication for general anesthesia in captive T. ornatus. However, higher dosages may be necessary under conditions of fieldwork [2]. Because general anesthesia was promptly induced after chemical immobilization, the total time of workable immobilization was unknown.

As opposed to bilateral motor blockade of the pelvic limbs produced by neuraxial anesthesia (e.g., epidural anesthesia), wildlife can benefit from unilateral blockade of the pelvic limbs produced by regional techniques because animals are able to rapidly regain standing position, reducing the stress during early postoperative period [8]. The ScFeNB technique was executed because of its indications for perioperative analgesia and isoflurane-sparing effects. The trend for an increase in HR and arterial blood pressure after the first hour of surgery (Case 1) could have been triggered by the transiently inadvertent intra-articular position of the intramedullary pin at the stifle joint. The innervation of the canine stifle involves multiple nerve components (e.g., sciatic, femoral, obturator, lateral femoral cutaneous nerves), therefore the ScFeNB alone might have been insufficient to induce complete desensitization of the stifle joint [9]. Another possibility is that the sciatic nerve was only partially blocked in view of the thick layer of adipose tissue of the thigh. Furthermore, the technique was oriented by guidelines available for small animals [23], while anatomical structures and landmarks may vary among species. Nevertheless, the ultrasound-guided $\mathrm{ScFeNB}$ showed promising results as 
Table 2. Range of heart rate (HR), arterial blood pressure (systolic [SAP], diastolic [DAP] and mean [MAP]), end-tidal carbon dioxide (ETCO $)_{2}$, end-tidal isoflurane $\left(\mathrm{ET}_{\mathrm{ISO}}\right)$ and peripheral oxygen saturation of hemoglobin $\left(\mathrm{SpO}_{2}\right)$ recorded in two isoflurane-anesthetized spectacled bears under mechanical ventilation.

\begin{tabular}{cccc}
\hline Variable & Bear 1 $(<1 \mathrm{~h})$ & Bear 1 $(>1 \mathrm{~h})$ & Bear 2 \\
\hline HR (beats/min) & $65-75$ & $85-105$ & $65-75$ \\
SAP (mmHg) & $90-105$ & $120-175$ & $85-110$ \\
DAP (mmHg) & $60-75$ & $75-110$ & $50-65$ \\
MAP (mmHg) & $67-80$ & $85-135$ & $63-77$ \\
ETCO $_{2}(\mathrm{mmHg})$ & $33-40$ & $38-48$ & $30-35$ \\
$\mathrm{ET}_{\mathrm{ISO}}(\%)$ & $1.3-1.9$ & $1.9-2.6$ & $1.1-1.7$ \\
$\mathrm{SpO}_{2}(\%)$ & $>90$ & $>90$ & $>90$ \\
\hline
\end{tabular}

most of the surgical procedure was accomplished under hemodynamic stability and low isoflurane requirements. To the authors' knowledge, this is the first description of the applicability of ultrasound guided regional anesthesia in a bear.

Hypertension and bradycardia are common findings during early phases of anesthesia with tiletamine-zolazepam combined with alpha ${ }_{2}$-agonist agents in ursids $[10,25]$. Transient hypertension is caused by increases in peripheral vascular tone, leading to baroreceptor mediated decreases in HR and cardiac output [17]. However, MAP remained within normal ranges for the most part of anesthesia (between 60 and $100 \mathrm{mmHg}$ ) (Table 2) and dexmedetomidine-induced hypertension was not detected during the first hour of maintenance of anesthesia with isoflurane because the alpha-2 agonist-related vasoconstrition is transient and isoflurane has vasodilating properties. When hypertension (MAP > $100 \mathrm{mmHg}$ ) was observed after 1 hour of anesthesia in Case 1, it was likely because of sympathetic stimulation caused by surgical stimulation.

Although direct arterial blood pressure measurements have been described for bears [2,25], arterial lines are usually placed in the femoral artery, which poses an inherent risk for bleeding and hematoma formation. Catheterization of the dorsal pedal artery is common practice in small animal anesthesia [14] and appeared to be a feasible option for spectacled bears.

It is challenging to determine whether the chemical restraint protocol caused bradycadia given the absence of physiological HR values for Tremarctos ornatus. Dexmedetomidine-premedicated captive
Asiatic black bears showed similar HR values ( $66 \mathrm{bpm}$ ) [7] in comparison to the spectacled bears of this report (70 bpm), whereas grizzly bears are considered to present bradycardia at HR below $50 \mathrm{bpm}$ [10]. Spectacled bears under tiletamine-zolazepam-based protocols also exhibited HR values in a similar range to that observed in the present report (64 - $71 \mathrm{bpm})[20]$. One should note that cardiovascular variables were first recorded approximately $50 \mathrm{~min}$ following initial darting. Therefore, it is likely that the effects of dexmedetomidine had weaned off considering its plasma half-life in dogs [16]. For this reason, the typical bradycardia and hypertension observed during early stages of the alpha2-agonist sedation might have been unnoticed.

Hypoxemia $\left(\mathrm{PaO}_{2}<80 \mathrm{mmHg}\right)$ is the most common complication in bear anesthesia under field conditions, where oxygen supplementation could be limited $[2,7,12,20,24]$. In order to evaluate oxygenation, not only $\mathrm{SpO}_{2}$ and $\mathrm{PaO}_{2}$ should be assessed, but also the $\mathrm{PaO}_{2} / \mathrm{FiO}_{2}$ ratio. Under normal conditions, the $\mathrm{PaO}_{2} / \mathrm{FiO}_{2}$ ratio should be above $400 \mathrm{mmHg}$ [18]. Despite the fact that both bears exhibited impaired oxygenation at the time of the first arterial blood gas sample, the $\mathrm{PaO}_{2} / \mathrm{FiO}_{2}$ ratio indicated an important oxygenation impairment only in Case 1, while in Case 2 the oxygenation was only moderately compromised. An arterial blood sample in Case 1 was not drawn when the animal was breathing room air before the onset of general anesthesia; yet a severe hypoxemia $\left(\mathrm{PaO}_{2}\right.$ $42 \mathrm{mmHg}$ ) was observed a week later when the bear was immobilized by the same drug combination while breathing room air (Table 1). 
Considering that $\mathrm{PaCO}_{2}$ was within normal ranges (Table 2), hypoventilation had no impact on oxygen exchange. For this reason, it was decided to perform an alveolar recruitment maneuver in both bears, following recommendations in horses under atelectasis-related hypoxemia [21]. In fact, right-to-left pulmonary shunt is an important source of oxygenation impairment in anesthetized horses [19]. Spectacled bears are medium-sized animals (approximately $100 \mathrm{~kg}$ ) and intrapulmonary shunting due to atelectasis related to recumbency could have contributed to impaired oxygenation, since the $\mathrm{PaO}_{2} / \mathrm{FiO}_{2}$ ratio was increased after the recruitment maneuver (Case 1). In light of the potential risks for alveolar collapse and perpetuation of intrapulmonary shunt when providing $100 \%$ oxygen due to resorption atelectasis [19], the $\mathrm{FiO}_{2}$ was lowered to 0.6 after an improvement in $\mathrm{PaO}_{2} / \mathrm{FiO}_{2}$ ratio was observed.

Given the paucity of information on spectacled bear anesthesia, this case report should aid wildlife veterinarians in the anesthetic management of such species. The dosages of drugs used in the present report may be a reference for chemical restraint in Tremarctos ornatus, as effective dosages may vary among species $[2,3]$. The need for close cardiorespiratory and arterial blood gas monitoring should be emphasized. Ultimately, oxygen supplementation and ventilatory support should be available to counteract hypoxemia.

\section{MANUFACTURERS}

${ }^{1}$ Virbac do Brasil Indústria e Comércio Ltda. São Paulo, SP, Brazil.

${ }^{2}$ Zoetis Indústria de Produtos Veterinários Ltda. Campinas, SP,

Brazil.

${ }^{3}$ Becton Dickinson Indústrias Cirúrgicas Ltda. Juiz de Fora, MG, Brazil.

${ }^{4}$ União Química Farmacêutica Nacional S.A. Embu-Guaçu, SP, Brazil.

${ }^{5}$ Lamedid Comercial e Serviços Ltda. São Paulo, SP, Brazil.

${ }^{6}$ Cristália Produtos Químicos Farmacêuticos Ltda. Itapira, SP,

Brazil.

${ }^{7}$ Mallard Medical Supply LLC. Irvine, CA, USA.

${ }^{8}$ JP Indústria Farmacêutica S.A. Ribeirão Preto, SP, Brazil.

${ }^{9}$ Digicare Biomedical. Boynton Beach, FL, USA.

${ }^{10}$ Radiometer Medical ApS. Brønshøj, Copenhagen, Denmark.

${ }^{11}$ GE Healthcare. Chicago, IL, USA.

${ }^{12}$ Mallinckrodt Pharmaceuticals. Staines-upon-Thames, SY, UK.

${ }^{13}$ Edwards Lifesciences. Irvine, CA, USA.

${ }^{14}$ Esaote Spa. Genoa, Italy.

${ }^{15}$ Hypofarma Instituto de Hypodermia e Farmácia Ltda. Ribeirão das Neves, MG, Brazil.

${ }^{16}$ Eurofarma Laboratórios S.A. São Paulo, SP, Brazil.

Declaration of interest. The authors report no conflicts of interest. The authors alone are responsible for the content and writing of the paper.

\section{REFERENCES}

1 Bouts T., Taylor P., Berry K., Routh A. \& Gasthuys F. 2011. Evaluation of medetomidine-ketamine and dexmedetomidine-ketamine in Chinese water deer (Hydropotes inermis). Veterinary Anaesthesia and Analgesia. 38(2): 106-102.

2 Cattet M.R.L., Caulkett N.A. \& Lunn N.J. 2003. Anesthesia of polar bears using xylazine-zolazepam-tiletamine or zolazepam-tiletamine. Journal of Wildlife Diseases. 39(3): 655-664.

3 Cattet M.R.L., Caulkett N. \& Stenhouse G.B. 2003. Anesthesia of grizzly bears using xylazine-zolazepam-tiletamine or zolazepam-tiletamine. Ursus. 14(1): 88-93.

4 Caulkett N. \& Cattet M.R.L. 1997. Physiological effects of medetomidine-zolazepam-tiletamine immobilization in black bears (Ursus americanus). Journal of Wildife Diseases. 33(3): 618-622.

5 Caulkett N. \& Cattet M.R.L. 2002. Anesthesia of Bears. In: Heard D. (Ed). Zoological Restraint and Anesthesia. Ithaca: International Veterinary Information Service, 6p.

6 Caulkett N. \& Fahlman A. 2014. Ursids (Bears). In: West G., Heard D. \& Caulkett N. (Eds). Zoo Animal and Wildlife Immobilization and Anesthesia. 2nd edn. Hoboken: John Wiley \& Sons, pp.599-606.

7 Coltrane J.A., Farley S., Saalfeld D., Battle D., Carnahan T. \& Teisberg J. 2015. Evaluation of Dexmedetomidine, Tiletamine, and Zolazepam for the Immobilization of Black Bears. Wildlife Society Bulletin. 39(2): 378-382.

8 D’Ovidio D., Noviello E. \& Adami C. 2016. Nerve stimulator-guided sciatic-femoral nerve block in raptors undergoing surgical treatment of pododermatitis. Veterinary Anaesthesia and Analgesia. 42(4): 449-453.

9 Echeverry D.F., Laredo F.G., Gil F., Belda E., Soler M. \& Agut A. 2012. Ultrasound-guided 'two-in-one' femoral and obturator nerve block in the dog: an anatomical study. Veterinary Anaesthesia and Analgesia. 39(6): 611-617.

10 Esteruelas N.F., Cattet M., Zedrosser A., Stenhouse G.B., Küker S., Evans A.L. \& Arnemo J.M. 2017. Double-Blinded, Randomized Comparison of Medetomidine-Tiletamine-Zolazepam and Dexmedetomidine-Tiletamine-Zolazepam Anesthesia in Free-Ranging Brown Bears (Ursus arctos). Plos One. 12(1): 1-23. 
11 Fahlman A., Arnemo J.M., Pringle J. \& Nyman G. 2014. Oxygen supplementation in anesthetized brown bears (Ursus arctos) - How low can you go? Journal of Wildlife Diseases. 50(3): 574-581.

12 Fahlman A., Arnemo J.M., Swenson J.E., Pringle J., Brunberg S. \& Nyman G. 2011. Physiologic evaluation of capture and anesthesia with medetomidine-zolazepam-tiletamine in brown bears (Ursus arctos). Journal of Zoo and Wildlife Medicine. 42(1): 1-11.

13 Fahlman A., Pringle J., Arnemo J.M., Swenson J.E., Brunberg S. \& Nyman G. 2010. Treatment of hypoxemia during anesthesia of brown bears (Ursus arctos). Journal of Zoo and Wildlife Medicine. 41(1): 161-164.

14 Garofalo N.A., Teixeira Neto F.J., Alvaides R.K., Oliveira F.A., Pignaton W. \& Pinheiro R.T. 2012. Agreement between direct, oscillometric and Doppler ultrasound blood pressures using three different cuff positions in anesthetized dogs. Veterinary Anaesthesia and Analgesia. 39(4): 324-334.

15 Jeong D., Yang J., Seok S., Song D. \& Yeon S. 2016. Cardiorespiratory effects of isoflurane in Asiatic black bears (Ursus thibetanus) anesthetized with intramuscular medetomidine and zolazepam/tiletamine. The Journal of Veterinary Medical Science. 79(1): 153-159.

16 Kuusela E., Raekallio M., Anttila M., Falck I., Mölsäs S. \& Vainio O. 2000. Clinical effects and pharmacokinetics of medetomidine and its enantiomers in dogs. Journal of Veterinary Pharmacology and Therapeutics. 23(1): 15-20.

17 Lemke K.A. 2004. Perioperative use of selective alpha- 2 agonists and antagonists in small animals. The Canadian Veterinary Journal. 45(6): 475-480.

18 Lopes P.C.F., Nunes N., Sousa M.G., Nishimori C.T., Carareto R., Santos P.S.P \& Camacho A.A. 2013. The effects of different inspired oxygen fractions on gas exchange and Tei-index of myocardial performance in propofol-anesthetized dogs. Veterinary Anaesthesia and Analgesia. 40(6): 573-583.

19 Marntell S., Nyman G. \& Hedenstierna G. 2005. High inspired oxygen concentrations increase intrapulmonary shunt in anaesthetized horses. Veterinary Anaesthesia and Analgesia. 32(6): 338-347.

20 McEntire M.S., Hope K.L., Hayek L.C. \& Siegal-Willott J.L. 2020. Review of anesthetic protocols in Andean bears (Tremarctos ornatus), sloth bears (Melursus ursinus), and giant pandas (Ailuropoda melanoleuca) at the Smithsonian Institution's National Zoological Park, 1995-2016. Journal of Zoo and Wildlife Medicine. 51(1): 67-79.

21 Moens Y. 2013. Mechanical Ventilation and Respiratory Mechanics During Equine Anesthesia. Veterinary Clinics of North America: Equine Practice. 29(1): 51-67.

22 Onuma M. 2003. Immobilization of sun bears (Helarctos malayanus) with medetomidine-zolezepam-tiletamine. Journal of Zoo and Wildlife Medicine. 34(2): 202-205.

23 Portela D.A., Verdier N. \& Otero P.E. 2018. Regional anesthetic techniques for the pelvic limb and abdominal wall in small animals: A review of the literature and technique description. The Veterinary Journal. 238: 27-40.

24 Romagnoli N., Pagnanelli G., Lambertini C., Drayton E., Buonacucina A. \& Peli A. 2018. Cardiorespiratory effets of medetomidine and dexmedetomidine combined with tiletamine-zolazepam for the immobilization of Asiatic black bears (Ursus thibetanus) under isoflurane general anesthesia. Plos One. 13(7): 1-9.

25 Teisberg J.E., Farley S.D., Nelson O.L., Hilderbrand G.V., Madel M.J., Owen P.A., Erlenbach J.A. \& Robbins C.T. 2014. Immobilization of grizzly bears (Ursus arctos) with dexmedetomidine, tiletamine and zolazepam. Journal of Wildlife Diseases. 50(1): 74-83.

26 The IUCN Red List of Threatened Species. 2017. Tremarctos ornatus. Available at $<$ https://www.iucnredlist.org/ species/22066/123792952> 